\title{
Dendritic Calcium Accumulation Associated With Direction-Selective Adaptation in Visual Motion-Sensitive Neurons In Vivo
}

\author{
RAFAEL KURTZ, VOLKER DÜRR, AND MARTIN EGELHAAF \\ Lehrstuhl für Neurobiologie, Fakultät für Biologie, Universität Bielefeld, D-33501 Bielefeld, Germany
}

Received 12 January 2000; accepted in final form 6 July 2000

\begin{abstract}
Kurtz, Rafael, Volker Dürr, and Martin Egelhaaf. Dendritic calcium accumulation associated with direction-selective adaptation in visual motion-sensitive neurons in vivo. J Neurophysiol 84: 1914-1923, 2000. Motion adaptation in directionally selective tangential cells (TC) of the fly visual system has previously been explained as a presynaptic mechanism. Based on the observation that adaptation is in part direction selective, which is not accounted for by the former models of motion adaptation, we investigated whether physiological changes located in the TC dendrite can contribute to motion adaptation. Visual motion in the neuron's preferred direction (PD) induced stronger adaptation than motion in the opposite direction and was followed by an afterhyperpolarization (AHP). The AHP subsides in the same time as adaptation recovers. By combining in vivo calcium fluorescence imaging with intracellular recording, we show that dendritic calcium accumulation following motion in the PD is correlated with the AHP. These results are consistent with a calcium-dependent physiological change in TCs underlying adaptation during continuous stimulation with PD motion, expressing itself as an AHP after the stimulus stops. However, direction selectivity of adaptation is probably not solely related to a calcium-dependent mechanism because direction-selective effects can also be observed for fast moving stimuli, which do not induce sizeable calcium accumulation. In addition, a comparison of two classes of TCs revealed differences in the relationship of calcium accumulation and AHP when the stimulus velocity was varied. Thus the potential role of calcium in motion adaptation depends on stimulation parameters and cell class.
\end{abstract}

\section{N T R O D U C T I O N}

Many sensory receptors and neurons adapt to a constant input or to repeated stimulation. Adaptation leads to a gradual decrease of response amplitude with time. This can reflect a shift in the operating range of the cell. Sensory systems thus retain their ability to detect small deviations of stimulus intensity from the adapting background level over a large range of stimulus intensities.

Decreased responsiveness on prolonged exposure to moving stimuli has been demonstrated in visual motion-sensitive neurons of insects and mammals (e.g., rabbit: Barlow and Hill 1963; cat: Vautin and Berkley 1977; fly: Maddess and Laughlin 1985; monkey: Petersen et al. 1985; butterfly: Maddess et al. 1991; wallaby: Ibbotson et al. 1998). Increased sensitivity to changes in velocity around an adapting level has been observed psychophysically in humans (Clifford and Langley 1996b;

Address for reprint requests: R. Kurtz, Lehrstuhl für Neurobiologie, Fakultät für Biologie, Universität Bielefeld, Postfach 1001 31, D-33501 Bielefeld, Germany (E-mail: rafael.kurtz@biologie.uni-bielefeld.de).
Clifford and Wenderoth 1999) and electrophysiologically in the fly's H1 cell (Jian and Horridge 1991; Maddess and Laughlin 1985). This neuron is 1 of more than 30 motion-sensitive tangential cells (TCs), which have been individually identified in the lobula plate, a part of the third visual neuropil (review articles: Egelhaaf and Borst 1993a; Hausen 1984; Hausen and Egelhaaf 1989). Movement detection is thought to be based on correlation of input from one retinal location with temporally delayed input from a neighboring location. A fully directionselective element, being excited by motion in the preferred direction (PD) and inhibited by motion in the opposite direction ("null direction," ND), is obtained by subtracting the output signals of such a correlator from that of its mirrorsymmetrical copy. This is called an "elementary motion detector," EMD (review articles: Borst and Egelhaaf 1989; Egelhaaf and Borst 1993b; Srinivasan et al. 1999). The vast dendrites of TCs are assumed to receive retinotopically arranged input from numerous local EMDs, thus being able to respond to certain types of optic flow in a large part of the visual field (Hausen 1981; Krapp and Hengstenberg 1996).

In the $\mathrm{H} 1$ neuron, motion adaptation remains restricted to that part of the receptive field in which an adapting stimulus was presented (Maddess and Laughlin 1985). Since no cell intrinsic local mechanism was known then, the associated physiological changes were thought to be located presynaptic to H1. Shortening of the EMD filter time constant, and thus tuning the motion detector to higher velocities, was proposed to underlie motion adaptation (Clifford and Langley 1996a; de Ruyter van Steveninck et al. 1986). A recent investigation on another class of TC, the horizontal system (HS) cells (Harris et al. 1999a), casts doubt on this model of motion adaptation: prolonged movement did not cause changes in the optimal velocity tuning as would be expected from adaptation of the EMD filter time constant. Therefore the physiological mechanism leading to motion adaptation and its location is still unclear.

In this study, we direct our attention to intrinsic physiological changes in the motion-sensitive TCs induced by prolonged stimulation. More specifically, we focus on a possible functional role of intracellular $\mathrm{Ca}^{2+}$. Because in many receptor cells and neurons the intracellular $\mathrm{Ca}^{2+}$ concentration affects cellular excitability, e.g., by regulating $\mathrm{Ca}^{2+}$ dependent conductances (review articles: neurons, Sah 1996; photoreceptors,

\footnotetext{
The costs of publication of this article were defrayed in part by the payment of page charges. The article must therefore be hereby marked "advertisement" in accordance with 18 U.S.C. Section 1734 solely to indicate this fact.
} 
Koutalos and Yau 1996; olfactory receptors, Menini 1999; hair cells, Fettiplace and Fuchs 1999), we investigate the relationship between adaptation and $\mathrm{Ca}^{2+}$ concentration changes and motion adaptation in TCs. Motion in restricted parts of the receptive field has been shown to lead to local $\mathrm{Ca}^{2+}$ accumulation, which remains restricted to the part of the dendrite getting input from the stimulated region of the receptive field (Borst and Egelhaaf 1992; Dürr and Egelhaaf 1999; Egelhaaf and Borst 1995). Hence, a $\mathrm{Ca}^{2+}$-based mechanism of motion adaptation in the TC could, similar to the formerly proposed presynaptic mechanism, account for the localized adaptive characteristics.

$\mathrm{A} \mathrm{Ca}^{2+}$-based mechanism would further imply that motion adaptation is direction selective. This expectation results from the observation, that major increases in dendritic $\mathrm{Ca}^{2+}$ concentration of TCs are only elicited by motion in the PD but not in the ND (Dürr 1998; Egelhaaf and Borst 1995). However, motion adaptation has not been analyzed before with test stimuli moving in the ND because former studies were mainly based on extracellular recordings of $\mathrm{H} 1$, which ceases spiking during motion in the ND. In our study, motion adaptation is tested both in the PD and in the ND by recording intracellularly from HS cells, in which graded membrane-potential changes can be recorded even in the axon (Hausen 1976, 1982a,b). We investigate the relation of calcium concentration changes to the electrical response characteristics underlying adaptation in this cell class and, additionally, in another class of TC, the centrifugal horizontal $(\mathrm{CH})$ cells (Eckert and Dvorak 1983; Egelhaaf et al. 1993; Hausen 1976). The latter cell class is supposed to possess an input organization similar to HS cells but to differ with respect to its output organization: its dendrites do not only form postsynaptic sites to EMDs but also dendritic presynaptic sites (Gauck et al. 1997) mediating inhibition to other TCs within the lobula plate (Warzecha et al. 1993). Therefore in the $\mathrm{CH}$ cell, $\mathrm{Ca}^{2+}$ might not only be relevant for modulatory functions but also for the control of transmitter release.

\section{METHOD S}

Electrical and optical recordings were performed in vivo from the three HS cells (Hausen 1982a,b) and the two CH cells (Eckert and Dvorak 1983; Egelhaaf et al. 1993; Hausen 1976) in female blowflies (Calliphora erythrocephala), aged 1-3 days. Data were pooled for each cell class because the major difference within a class is the receptive field location with known physiological differences being only of minor significance (Eckert and Dvorak 1983; Hausen 1982a,b). For information about the animal preparation and closer details concerning the further experimental procedures, see Dürr and Egelhaaf (1999). Data are presented as means \pm SE.

\section{Electrophysiology}

Ringer solution (containing, in $\mathrm{mM}, 128.3 \mathrm{NaCl}, 5.4 \mathrm{KCl}, 1.9$ $\mathrm{CaCl}_{2}, 4.8 \mathrm{NaHCO}_{3}, 3.3 \mathrm{Na}_{2} \mathrm{HPO}_{4}, 3.4 \mathrm{KH}_{2} \mathrm{PO}_{4}, 13.9$ glucose, $\mathrm{pH}$ 7.0; all chemicals from Merck) was used to prevent desiccation of the recording region and for filling a wide-tip glass pipette used as indifferent electrode. Electrodes for intracellular recording were made from borosilicate glass (GC100F-10, Clark Electromedical) on a Brown-Flaming puller (P97, Sutter Instruments) and had resistances of 20-40 M $\Omega$ when filled with $1 \mathrm{M} \mathrm{KCl}$. Voltage records (Axoclamp $2 \mathrm{~A}$, Axon Instruments) were low-pass filtered at $2 \mathrm{kHz}$, and AID converted at a sampling rate of $1 \mathrm{kHz}$ with an amplitude resolution of $0.0244 \mathrm{mV}$ (DT2801A, Data Translation). Part of the recordings were performed during iontophoretic injection of the fluorescent dye by a weak tonic hyperpolarizing current (see following text). No significant differences in the electrical properties were observed between the cells hyperpolarized in this way and the cells recorded without current injection.

\section{Imaging changes in intracellular $\mathrm{Ca}^{2+}$ concentration}

The calcium-sensitive dye Fura-2 was injected iontophoretically into single neurons by a hyperpolarizing current of $0.8 \mathrm{nA}$, with electrodes containing (in $\mathrm{mM}$ ) $33.3 \mathrm{KCl}$ (Sigma), $1.7 \mathrm{KOH}$ (Merck), 20.0 fura-2 pentapotassium salt (Molecular Probes), and 33.3 HEPES (Sigma), pH 7.3. Relative changes of intracellular ionic free $\mathrm{Ca}^{2+}$ concentration $\left(\Delta\left[\mathrm{Ca}^{2+}\right]_{\mathrm{i}}\right)$ were determined during or immediately following electrophysiological recording by a single wavelength measurement according to Vranesic and Knöpfel (1991). Epifluorescence measurements were performed under an upright microscope (Universal III-RS, Zeiss). Fluorescence was elicited at $380 \mathrm{~nm}$ (light emitted from a Hg arc lamp, HBO 100W, Osram, and band-pass filtered with a bandwidth of $10 \mathrm{~nm}$ ) and measured after passage of a 410-nm dichroic mirror and a 500- to 530-nm band-pass filter. Images were magnified 20-fold by the microscope objective (UD20, Zeiss) and acquired with a CCD camera (PXL, Photometrics) controlled by PMIS software (GKR Computer Consulting). Acquisition rate was approximately $3 \mathrm{~Hz}$ and spatial resolution was $9 \mu \mathrm{m} . \Delta F / F$ was determined relative to an image obtained prior to visual stimulation. The values were averaged in a region covering the entire dendritic tree. Increases in $\left[\mathrm{Ca}^{2+}\right]_{\mathrm{i}}$ lead to a decrement in fluorescence. For convenience when comparing the calcium signals with the electrical signals of a cell, the former were inverted in the figures. Increments in $\left[\mathrm{Ca}^{2+}\right]_{\mathrm{i}}$ are thus indicated by positive $\Delta F / F$ values. Background fluorescence as determined for each image in a region surrounding the dendrite was subtracted from the fluorescence images.

\section{Visual stimuli}

TCs were stimulated by two stripe patterns projected through a circular aperture on a translucent hemisphere and moving horizontally in the cells' receptive field. The stimulus was presented ipsilaterally and was characterized by the following parameters: diameter, $66^{\circ}$; spatial wavelength, ca. $33^{\circ}$; contrast, 0.68 ; mean light intensity, $12 \mathrm{~cd}$ $\mathrm{m}^{-2}$ with a spectral composition of a $\mathrm{Hg}$ arc lamp (HBO $100 \mathrm{~W}$, Osram) filtered by a blue filter (BG1, Schott). The stimulus velocity depended on the protocol and is given in the text and figure legends as temporal frequency (cycles per second; $2-\mathrm{Hz}$ temporal frequency thus corresponds to an angular velocity of $66^{\circ} \mathrm{s}^{-1}$ ).

\section{R E S U L T S}

\section{Adaptation of TC responses}

The HS and $\mathrm{CH}$ cells of the fly respond to visual motion with graded membrane potential changes $\left(\Delta E_{\mathrm{M}}\right)$. In HS cells, depolarizing responses are usually superimposed by spike-like rapid depolarizations (Hausen 1982a). When a stripe pattern in the receptive field starts to move at a constant velocity in the PD of the cell, the membrane potential of both HS and CH cells rapidly shifts to a depolarized state followed by a slow decline to a steady-state level (Fig. 1). This transient behavior can, in part, be attributed to the mechanism of motion detection itself (Egelhaaf and Borst 1989) but additionally reflects changes in the movement processing system dependent on stimulus history, i.e., motion adaptation (Maddess and Laughlin 1985). The influence of stimulus history becomes obvious if a test stimulus is preceded by an adapting stimulus: if the interval between these two stimuli is sufficiently short (e.g., $0.1 \mathrm{~s}$, as in Fig. 1), 


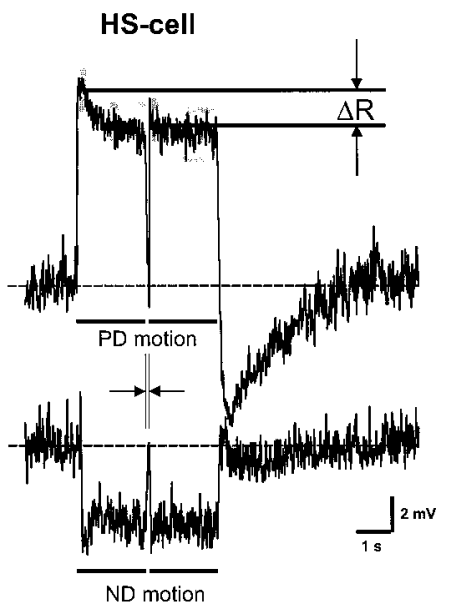

CH-cell
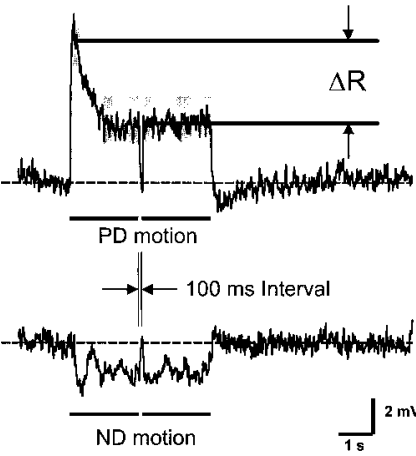

FIG. 1. Adaptation in the graded membrane potential responses of tangential cells (TCs). Membrane potential change $\left(\Delta E_{\mathrm{M}}\right)$ of a horizontal system cell (HS) (left) and a centrifugal horizontal $(\mathrm{CH})$ cell (right) relative to the resting level (- - -, determined in a 1-s period prior to stimulation) in response to visual motion stimuli (marked by underneath the response traces). An adapting stimulus was followed by an identical test stimulus, separated by an interval of $100 \mathrm{~ms}$. Stimulus motion lasted $2 \mathrm{~s}$, with $2-\mathrm{Hz}$ temporal frequency. Direction of motion was either in the preferred direction (PD, top) or in the null direction (ND, bottom) of the neuron. Individual traces, recorded from $1 \mathrm{HS}$ and $1 \mathrm{CH}$ cell, respectively, were averaged and smoothed off-line for presentation using a running average of $25 \mathrm{~ms}$ width. Time windows for calculation of the early response component (100-300 ms after stimulus onset) and the late response component (1-2 s after stimulus onset) are indicated by gray shading $(\square) . \Delta R$, denoting the difference between adapting and test response (indicated here for the early response components), was used as a measure of adaptation. the early component of the response to the test stimulus is attenuated, rendering the test response less transient than the adapting response. The latter can serve as reference in our experimental procedure (Fig. 1). Motion adaptation, measured as the attenuation of the early component of the test response, can also be observed in both $\mathrm{HS}$ and $\mathrm{CH}$ cells for stimuli moving in the ND.

\section{Direction selectivity of adaptation}

Adaptation is weaker and recovery from adaptation is faster for motion in the ND compared with the PD. This was shown quantitatively for HS cells by presenting adapting and test stimuli, separated by variable intervals. The experiments were done both for motion in the PD and in the ND (Fig. 2, left). To allow easier comparison of $\Delta E_{\mathrm{M}}$ in response to PD motion (top traces in each row), and $\Delta E_{\mathrm{M}}$ in response to ND motion (bottom traces in each row), ND traces are shown inverted and scaled by a factor of 1.5. Direction dependency is most prominent at the medium interval length $(0.5 \mathrm{~s})$, where the attenuation of the early component of the test response is pronounced for PD motion but hardly detectable for ND motion.

In Fig. $3 A$, the amplitudes of the early component (200-ms period, starting $100 \mathrm{~ms}$ after stimulus onset, see Fig. 1) of adapting and test responses are plotted for the PD and the ND. Adaptation is marked by a weaker early component of the response to the test than to the adapting stimulus. For motion in the PD, this amplitude reduction is significant for the 0.1and 0.5-s inter-stimulus intervals. No significant amplitude
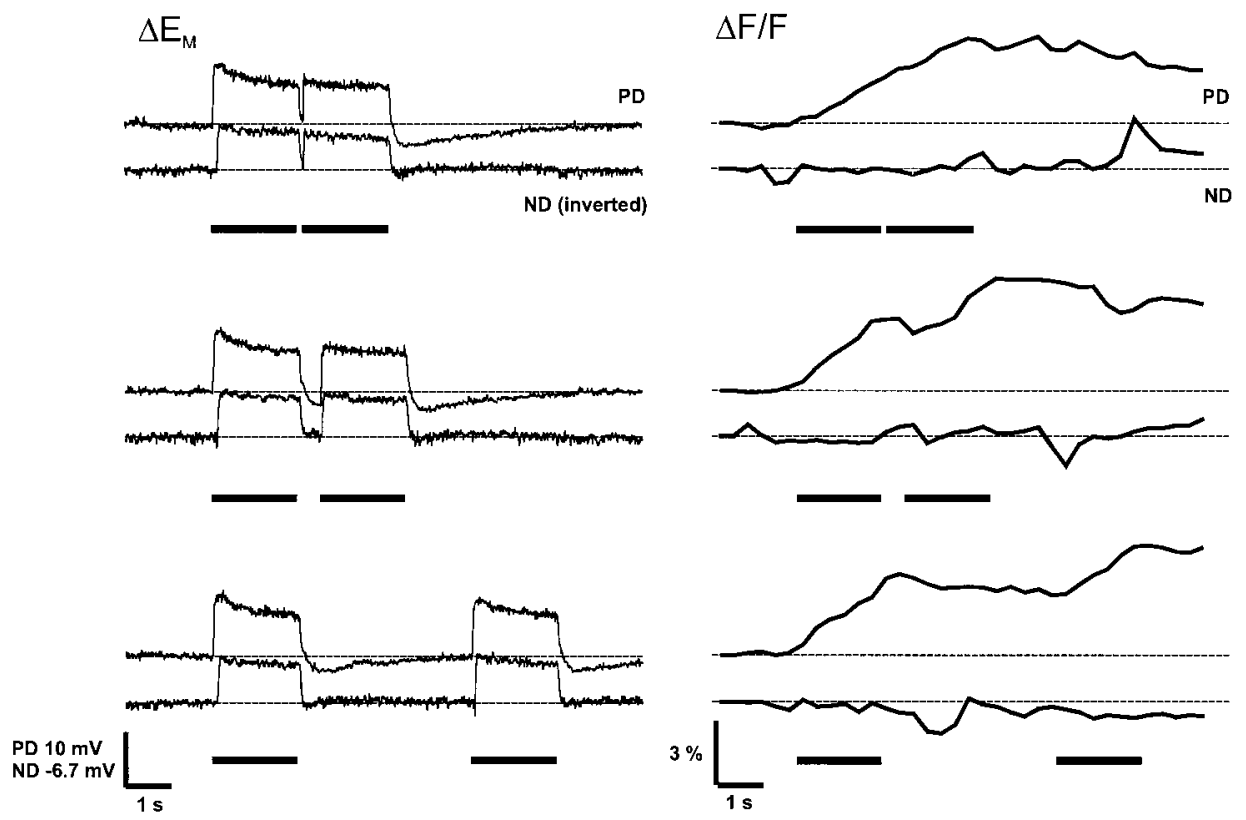

FIG. 2. Direction selectivity of adaptation and dendritic $\mathrm{Ca}^{2+}$ accumulation. $\Delta E_{\mathrm{M}}$ and concentration change of intracellular ionic free calcium $\left(\Delta\left[\mathrm{Ca}^{2+}\right]_{\mathrm{i}}\right)$ in response to adapting and test stimuli. Shown are averaged responses measured in vivo in HS cells with voltage and $\mathrm{Ca}^{2+}$ imaging performed simultaneously or with $\mathrm{Ca}^{2+}$ imaging performed independently using identical stimulation (the latter within about $0.5 \mathrm{~h}$ of voltage recordings). Stimulation protocol as in Fig. 1, except that 3 different inter-stimulus intervals were tested (top, $0.1 \mathrm{~s} ;$ middle, $0.5 \mathrm{~s} ;$ bottom, $4 \mathrm{~s}$; stimulation periods are marked by horizontal bars underneath the response traces). For easier comparison, $\Delta E_{\mathrm{M}}$ in response to ND motion (left, bottom traces in each row) is shown inverted and scaled by a factor of 1.5. Averaged time course of $\Delta\left[\mathrm{Ca}^{2+}\right]_{\mathrm{i}}$ (right) determined as relative fluorescence change $(\Delta F / F)$ at $\lambda=380 \mathrm{~nm}$ in areas covering the whole dendrite of HS cells filled with Fura 2. The horizontal line represents the baseline given by the first image, in relation to which $\Delta F / F$ in all subsequent images was calculated. As explained in METHODS, $\Delta F / F$ values are inverted, with positive $\Delta F / F$ corresponding to increments in $\left[\mathrm{Ca}^{2+}\right]_{\mathrm{i}}$. Unlike the $\Delta E_{\mathrm{M}}$ traces, $\Delta F / F$ traces of PD and ND stimulation are drawn with the same orientation and scale. Sample sizes: electrophysiological data: $n=12$ (same set of cells for PD and ND). Data on $\mathrm{Ca}^{2+}: n=$ 5-6 for PD and $n=3-4$ for ND. 
A

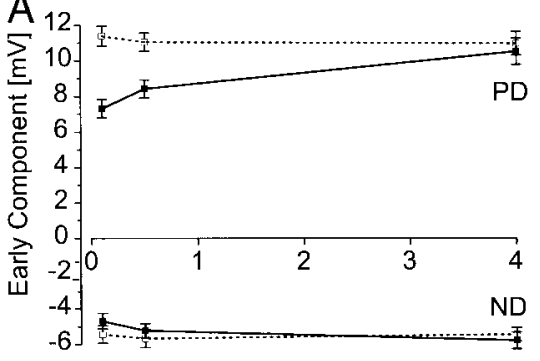

Time after $1^{\text {st }}$ stimulus [s]

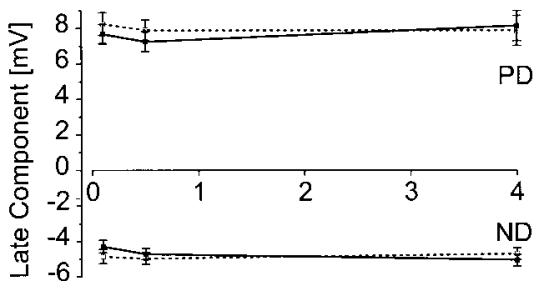

Time after $1^{\text {st }}$ stimulus [s]

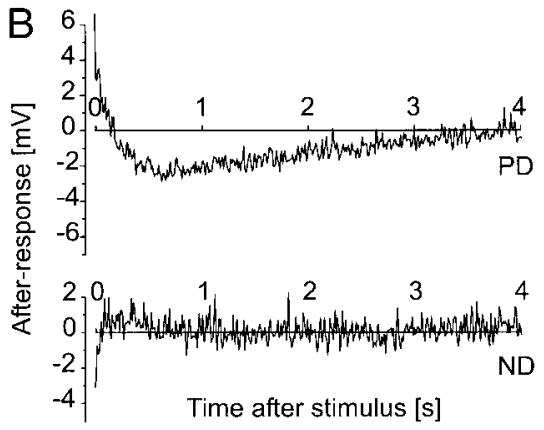

FIG. 3. Time course of recovery from adaptation and after-reactions following PD and ND motion. A: amplitude of the early component (top) and the late component (bottom) of $\Delta E_{\mathrm{M}}$ (for time windows, see Fig. 1) in response to adapting $(\bigcirc)$ and test stimuli $(\bullet)$, plotted as a function of inter-stimulus interval. Responses to adapting stimuli serve as reference. The strength of adaptation is thus indicated by the difference between adapting and test responses. Mean \pm SE of $12 \mathrm{HS}$ cells (same set of cells for PD and ND). $B$ : after-reactions recorded from HS cells (averaged responses from 10 cells) following a $2-\mathrm{Hz}$ motion stimulus of 2-s duration in PD (top) and ND (bottom). Time 0 marks the cessation of stimulus motion. Baseline voltage was determined in a 1-s period prior to stimulation. For presentation, traces were smoothed off-line using a running average of 10 -ms width.

difference between adapting and test response can be observed if both are separated by a 4-s interval. In each cell tested, $\Delta R$, i.e., the difference between the early component of the response to the adapting and to the test stimulus, respectively (see Fig. 1), is positive for both the 0.1 - and the 0.5 -s interstimulus interval (averaged values: $\Delta R_{\mathrm{PD}}=4.08 \pm 0.50 \mathrm{mV}$ for the 0.1 -s interval and $\Delta R_{\mathrm{PD}}=2.63 \pm 0.32 \mathrm{mV}$ for the $0.5-\mathrm{s}$ interval, $n=12$ ). Statistical significance of the difference between adapting response and test response was confirmed by a Wilcoxon rank test $(P<0.005$ for both 0.1 - and $0.5-\mathrm{s}$ inter-stimulus interval, $n=12$ ). In contrast, an adapting stimulus in the ND leads to an attenuation of the early component of the test response (leading to negative $\Delta R$ values) in only 8 of 12 cells for both the 0.1 - and the 0.5 -s interval. Averaged for all cells, the absolute values of $\Delta R$ for the $\mathrm{ND}\left(\Delta R_{\mathrm{ND}}=\right.$ $-0.76 \pm 0.40 \mathrm{mV}$ for the 0.1 -s interval and $\Delta R_{\mathrm{ND}}=-0.45 \pm$ $0.32 \mathrm{mV}$ for the 0.5 -s interval, $n=12$ ) are almost an order of magnitude lower than the corresponding values for the PD (see preceding results). Hence for ND motion, a statistical difference between adapting response and test response is given only for the 0.1-s interval at a low significance level (Wilcoxon rank test, $P<0.1, n=12$ ).

To assess the directional selectivity of adaptation, we determined the attenuation of the early response component by a relative measure ( $r=$ test response/adapting response). Ratios calculated for $\mathrm{PD}\left(r_{\mathrm{PD}}\right)$ and $\mathrm{ND}$ motion $\left(r_{\mathrm{ND}}\right)$, respectively, were statistically different both for the $0.1-$ and the $0.5-\mathrm{s}$ inter-stimulus interval. Thus adaptation contains a directionally selective component (0.1-s interval: $r_{\mathrm{PD}}=0.645 \pm 0.036$, $r_{\mathrm{ND}}=0.887 \pm 0.074 ; 0.5$-s interval: $r_{\mathrm{PD}}=0.762 \pm 0.028$, $r_{\mathrm{ND}}=0.970 \pm 0.085, \mathrm{H}_{0}: r_{\mathrm{PD}}=\mathrm{r}_{\mathrm{ND}}$, Wilcoxon rank test, $P<$ 0.05 in both cases, $n=12$ ).

Regardless of the direction of motion (PD or ND), the late component of the test response (last second of the 2-s stimulation period, see Fig. 1) is not greatly diminished by adaptation (Fig. 3A). Nevertheless, probably due to the better signalto-noise ratio resulting from the larger time window, for an inter-stimulus interval of either 0.1 or $0.5 \mathrm{~s}$, there is significant attenuation of the late response for PD and for ND motion (0.1-s interval: $\Delta R_{\mathrm{PD}}=0.57 \pm 0.23 \mathrm{mV}, \Delta R_{\mathrm{ND}}=-0.56 \pm$ $0.14 \mathrm{mV}$; 0.5 -s interval: $\Delta R_{\mathrm{PD}}=0.65 \pm 0.26 \mathrm{mV}, \Delta R_{\mathrm{ND}}=$ $-0.25 \pm 0.10 \mathrm{mV}$, Wilcoxon rank test, $P<0.05$ in all cases, $n=12$ ). No direction selectivity was found for adaptation of the late response components (0.1-s interval: $r_{\mathrm{PD}}=0.945 \pm$ $0.034, r_{\mathrm{ND}}=0.900 \pm 0.034 ; 0.5$-s interval: $r_{\mathrm{PD}}=0.923 \pm$ $0.034, r_{\mathrm{ND}}=0.953 \pm 0.020,4-\mathrm{s}$ interval: $r_{\mathrm{PD}}=1.106 \pm$ $0.107, r_{\mathrm{ND}}=1.091 \pm 0.044 ; \mathrm{H}_{0}: r_{\mathrm{PD}}=r_{\mathrm{ND}}$, Wilcoxon rank test, $P>0.05$ in all cases, $n=12$ ).

Since PD motion elicits, on average, stronger responses than ND motion, direction-selective effects could be mimicked by processes that are in fact nonlinearly dependent on response size. Therefore we also compared adaptation in HS cells with approximately equally sized PD and ND responses. As has been found for the whole cell sample, the attenuation of the early response component by adapting stimuli was more pronounced and longer lasting for PD motion than for ND motion in this subset of neurons. Thus adaptation in TCs has a genuine direction-selective component.

In conclusion, our results indicate that motion adaptation in HS cells is characterized by both direction-selective and nondirectional mechanisms. Direction selectivity is reflected in a more pronounced attenuation of the early component of the test response to PD motion compared with ND motion. Additionally, recovery from adaptation is faster for PD than for ND motion. Compared to direction-selective adaptation, the nondirectional component of motion adaptation is weak but does result in a statistically significant reduction of the late component of the test response.

Adaptation in $\mathrm{CH}$ cells, tested experimentally in the same way for a smaller cell sample, followed a similar pattern. This, however, does not preclude quantitative differences in the characteristics of adaptation between the two cell classes.

\section{After-reactions reflect motion adaptation in its strength and recovery time course}

After PD motion stops, the membrane potentials of HS and $\mathrm{CH}$ cells hyperpolarize, a phenomenon referred to here as afterhyperpolarization (AHP) (Figs. 1 and $3 B$ ). In contrast, 
there is a comparatively weak and more transient depolarizing after-reaction following ND motion. An attenuation of the early component of the test response is only detectable if the test response occurs while the after-reaction is still present (compare Fig. 3, $A$ and $B$ ). Likewise, the small adaptation amplitude and its fast recovery during motion in the ND is paralleled by a low amplitude and a fast recovery time course of the after-reaction following ND motion (see Fig. 3).

These results suggest, that direction-selective adaptation is based on a postsynaptic cellular mechanism in the TCs, for example an increased $\mathrm{K}^{+}$conductance or a decreased nonselective cation conductance, which on the one hand leads to a reduction in response strength during prolonged motion stimulation and on the other hand results in an AHP on stimulus cessation.

\section{Correlation of AHP with dendritic $\mathrm{Ca}^{2+}$ accumulation}

Motion stimulation in the PD has previously been shown to increase the $\mathrm{Ca}^{2+}$ concentration in TCs (Borst and Egelhaaf 1992; Dürr and Egelhaaf 1999; Egelhaaf and Borst 1995; Egelhaaf et al. 1993; Single and Borst 1998). By optical recording of fluorescence changes of the $\mathrm{Ca}^{2+}$ indicator fura-2 in the dendrites of $\mathrm{HS}$ and $\mathrm{CH}$ cells, we measured the relative changes in the intracellular $\mathrm{Ca}^{2+}$ concentration $\left(\Delta\left[\mathrm{Ca}^{2+}\right]_{\mathrm{i}}\right)$ in vivo during visual stimulation. HS cells showed significant rises in $\left[\mathrm{Ca}^{2+}\right]_{\mathrm{i}}$ in response to adapting and test stimuli moving in the PD but almost no changes for ND motion (Fig. 2). As was shown previously, $\Delta\left[\mathrm{Ca}^{2+}\right]_{\mathrm{i}}$ differs from $\Delta E_{\mathrm{M}}$ in its time course: The slope of $\Delta\left[\mathrm{Ca}^{2+}\right]_{\mathrm{i}}$ is less steep than that of $\Delta E_{\mathrm{M}}$ and continues to rise when the latter already reaches a steadystate plateau (Dürr and Egelhaaf 1999). Furthermore, whereas the decay of $\Delta E_{\mathrm{M}}$ after the stimulus stops takes place within tens or hundreds of milliseconds, the decay of $\left[\mathrm{Ca}^{2+}\right]_{\mathrm{i}}$ is characterized by time constants of several seconds.

To test a potential role of calcium in motion adaptation, we analyzed whether the strength of the AHP correlates with $\Delta\left[\mathrm{Ca}^{2+}\right]_{\mathrm{i}}$ in the dendrites of $\mathrm{HS}$ and $\mathrm{CH}$ cells. In response to PD motion stimuli of increasing duration, both cell classes show dendritic $\Delta\left[\mathrm{Ca}^{2+}\right]_{\mathrm{i}}$ with increasing peak amplitudes (Fig. 4A). The time course of both the increase in $\left[\mathrm{Ca}^{2+}\right]_{i}$ during motion stimulation and the decrease of $\left[\mathrm{Ca}^{2+}\right]_{i}$ after termination of stimulus motion is faster in $\mathrm{CH}$ than in $\mathrm{HS}$ cells as has been described earlier (Dürr and Egelhaaf 1999). During $4 \mathrm{~s}$ of ND motion (Fig. 4A, bottom), little deviation from baseline is seen in the mean $\Delta\left[\mathrm{Ca}^{2+}\right]_{\mathrm{i}}$. A systematic investigation revealed that $\mathrm{CH}$ but not $\mathrm{HS}$ cells show, on

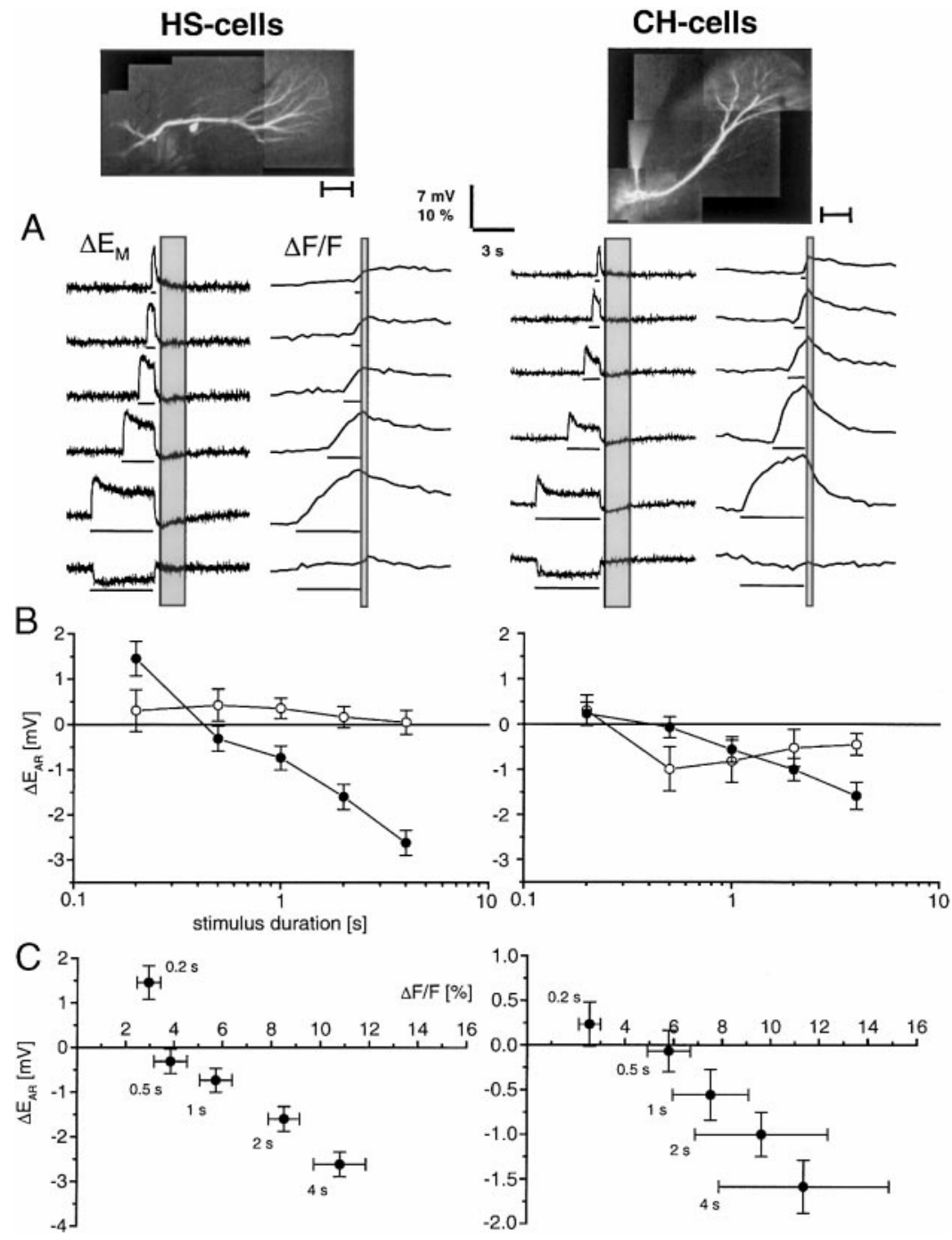

FIG. 4. Relationship of $\left[\mathrm{Ca}^{2+}\right]_{\mathrm{i}}$ and after-reaction of the membrane potential in response to motion of variable duration. Left: data for HS cells; right: data for $\mathrm{CH}$ cells. Photomontages constructed of raw fluorescence images of a southern cell of the horizontal system (HSS) and a dorsal centrifugal horizontal $(\mathrm{DCH})$ cell, recording electrode visible in the left part of the picture, shown on top left and right, respectively; scale bars $100 \mu \mathrm{m} . A$ : averaged $\Delta E_{\mathrm{M}}$ and $\Delta\left[\mathrm{Ca}^{2+}\right]_{\mathrm{i}}$ in response to visual motion stimuli of variable duration. Horizontal bars indicate period of stimulus motion (top to bottom: $200 \mathrm{~ms}, 500 \mathrm{~ms}, 1 \mathrm{~s}$, $2 \mathrm{~s}, 4 \mathrm{~s}$ in $\mathrm{PD}$ and $4 \mathrm{~s}$ in ND). Time course of $\Delta \mathrm{E}_{\mathrm{M}}$ (left part of each block) with shaded box, indicating time window for the calculation of the after-reaction ( $200 \mathrm{~ms}-2 \mathrm{~s}$ after stimulus cessation). Time course of $\Delta \mathrm{F} / \mathrm{F}$ (right part of each block) with shaded box, indicating single frame from which $\Delta \mathrm{F} / \mathrm{F}$ at the end of the motion stimulus was calculated. $B$ : After-reaction of the membrane potential $\left(\Delta \mathrm{E}_{\mathrm{AR}}\right)$ as a function of stimulus duration (plotted on a logarithmic scale). Mean values for stimulus motion in $\mathrm{PD}(\bullet)$ and in $\mathrm{ND}(\mathrm{O}) . C: \Delta \mathrm{E}_{\mathrm{AR}}$ following $\mathrm{PD}$ motion of variable duration (given beside each data point) as a function of $\Delta\left[\mathrm{Ca}^{2+}\right]_{\mathrm{i}}$. Note the different scaling of the ordinate for HS- and CH-cells. Sample sizes: Electrophysiological data: HS-cells, $n=15-16$ for PD and $n=7-15$ for ND; CH-cells, $n=7-8$ for PD and $n=5-6$ for ND. Data on $\mathrm{Ca}^{2+}$ : HS-cells, $n=5$; CH-cells, $n=4$. Error bars denote SE in all cases. 
average, decrements in dendritic $\left[\mathrm{Ca}^{2+}\right]_{\mathrm{i}}$ in response to ND motion (unpublished results).

After-reactions of the membrane potential were quantified in a time window of $1.8 \mathrm{~s}$, starting $0.2 \mathrm{~s}$ after the stimulus stopped. An AHP occurs only when PD motion lasts for at least $0.5 \mathrm{~s}$. If a shorter stimulus is presented, the depolarization decays rather slowly and the after-reaction is on a depolarized level (Fig. 4A). Plotting the amplitude of the after-reaction $\left(\Delta E_{\mathrm{AR}}\right)$ to $\mathrm{PD}$ motion versus the logarithm of stimulus duration reveals that for both cell classes $\Delta E_{\mathrm{AR}}$ steadily decreases with increasing stimulus duration (Fig. $4 B$ ). In contrast, for ND motion, HS cells show small after-depolarizations, the amplitudes of which do not depend on stimulus duration. Although $\mathrm{CH}$ cells show hyperpolarizing after-reactions to ND motion, again, there is no monotonic dependency of $\Delta E_{\mathrm{AR}}$ on stimulus duration.

Finally, there is a monotonic relationship between AHP and $\mathrm{Ca}^{2+}$ accumulation for PD motion both for HS and $\mathrm{CH}$ cells. This is demonstrated by plotting $\Delta E_{\mathrm{AR}}$ as a function of the relative fluorescence change $\Delta F / F$ at the end of the motion stimulus (Fig. 4C).

\section{Dependence on stimulus velocity of $\mathrm{Ca}^{2+}$ accumulation and} motion adaptation

The response of visual motion-sensitive neurons such as HS and $\mathrm{CH}$ cells is not only determined by the duration of the stimulus but also by other stimulus parameters, such as velocity. Therefore we investigated whether a correlation between AHP and $\left[\mathrm{Ca}^{2+}\right]_{\mathrm{i}}$ also exists if the stimulus strength is altered by varying the velocity of PD motion. Due to the nature of motion detection, $\mathrm{HS}$ and $\mathrm{CH}$ cells show membrane-potential responses of maximal size at a certain temporal frequency, below and beyond which the amplitudes decline (Dürr 1998; Egelhaaf and Borst 1989; Hausen 1982b). In HS cells, the corresponding velocity tuning of $\Delta\left[\mathrm{Ca}^{2+}\right]_{\mathrm{i}}$ is known to follow a similar characteristic (Dürr 1998). In contrast, CH cells exhibit a discrepancy between the velocity tuning of the $\left[\mathrm{Ca}^{2+}\right]_{\mathrm{i}}$ response and that of the electrical response (Dürr 1998). In a plot of $\Delta E_{\mathrm{AR}}$ values versus $\Delta F / F$, both determined in the same way as for Fig. $4 C$, but for motion stimuli of constant duration varying in temporal frequency, a monotonic relationship between the two parameters is obtained for HS but not for $\mathrm{CH}$ cells (Fig. 5).

At very high velocities (24-Hz temporal frequency), HS cells respond with a distinct, though transient, membrane depolarization, which is accompanied by little or no $\left[\mathrm{Ca}^{2+}\right]_{\mathrm{i}}$

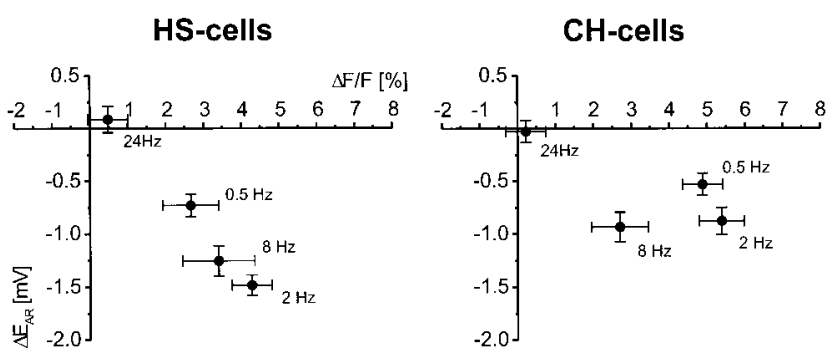

FIG. 5. Relationship of $\Delta\left[\mathrm{Ca}^{2+}\right]_{\mathrm{i}}$ and $\Delta E_{\mathrm{AR}}$ in response to PD motion of variable temporal frequency. Determination of $\Delta F / F$ and $\Delta E_{\mathrm{AR}}$ as in Fig. 4. Sample sizes: electrophysiological data: HS cells, $n=30$; $\mathrm{CH}$ cells, $n=$ 27-31. Data on $\mathrm{Ca}^{2+}$ : HS cells, $n=9-12$; CH cells, $n=10-12$. Error bars denote SE.
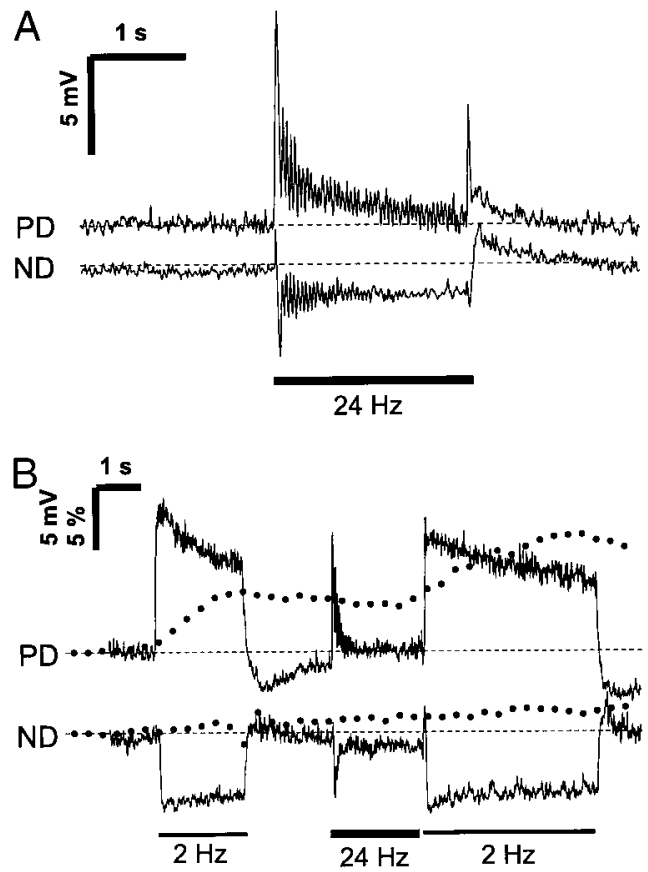

FIG. 6. Adaptation caused by stimuli moving at a high velocity. A: averaged $\Delta E_{\mathrm{M}}$ recorded from HS cells $(n=10)$ when a stimulus with a temporal frequency of $24 \mathrm{~Hz}$ and a duration of $2 \mathrm{~s}$ is presented either in PD (top) or ND (bottom). $B: \Delta E_{\mathrm{M}}(-)$ and $\Delta\left[\mathrm{Ca}^{2+}\right]_{\mathrm{i}}(\cdots)$ on presentation of a reference $2-\mathrm{Hz}$ stimulus, followed after $1.9 \mathrm{~s}$ by a $24-\mathrm{Hz}$ adapting stimulus and after an interval of $100 \mathrm{~ms}$ by a $2-\mathrm{Hz}$ test stimulus. Averaged time course recorded from HS cells (electrophysiological data: $n=5$; data on $\mathrm{Ca}^{2+}: n=4$ for PD and $n=3$ for ND).

increases (Dürr 1998). As expected if the AHP depends on cytosolic $\mathrm{Ca}^{2+}$, membrane potential changes induced by 24-Hz stimuli are not followed by an AHP (Fig. 6A). In contrast, an after-depolarization follows, comparable in strength and time course to the one seen after ND motion.

Although adaptation renders membrane potential responses more transient, the time course by itself cannot serve as an unequivocal indicator of adaptation because, inherent to the mechanism of motion detection, responses are more transient for fast stimuli than for slow stimuli (cf. Figs. 1 and 6A) (Egelhaaf and Borst 1989). However, differences in the time course of responses to PD and ND motion may indicate direction-selective adaptation. The responses to fast moving stimuli shown in Fig. 6A exhibit two direction-selective characteristics: first, the response to ND motion remains on a slightly larger steady-state membrane potential level than the PD response. Second, the after-reactions of responses to PD and ND motion are not mirror symmetrical, particularly because responses to both PD and ND motion are followed by afterdepolarizations. Since stimuli moving at a temporal frequency of $24 \mathrm{~Hz}$ were shown to cause at most very slight $\Delta\left[\mathrm{Ca}^{2+}\right]_{\mathrm{i}}$, $\mathrm{Ca}^{2+}$-dependent adaptation cannot be assumed to be the sole determinant of direction-selective effects.

\section{Direction-selective adaptation potentially independent of $\mathrm{Ca}^{2+}$ accumulation}

As outlined in the preceding paragraph, fast moving stimuli cause very transient membrane potential responses. During PD motion, these are accompanied by at most very small 
$\left[\mathrm{Ca}^{2+}\right]_{\mathrm{i}}$ increases (Dürr 1998). To assess the strength and direction selectivity of motion adaptation caused by a fast moving stimulus, hence without pronounced concurrent $\mathrm{Ca}^{2+}$ increase, HS cells were stimulated by the following protocol: First, $2-\mathrm{Hz}$ motion was presented for $2 \mathrm{~s}$ as a reference stimulus. After $1.9 \mathrm{~s}, 24-\mathrm{Hz}$ motion was presented for $2 \mathrm{~s}$ as the adapting stimulus, followed after $100 \mathrm{~ms}$ by a $2-\mathrm{Hz}$ test stimulus (Fig. 6B). During PD motion, the transient response to the test stimulus is attenuated relative to that elicited by the reference stimulus. The size of this effect renders it unlikely that it is completely due to the initial $2-\mathrm{Hz}$ stimulus because adaptation elicited by such a stimulus has recovered after an interval of $4 \mathrm{~s}$ (see Fig. 3A). Thus the attenuation is at least partially due to the $24-\mathrm{Hz}$ stimulus. The adapting effect of $24-\mathrm{Hz}$ motion is probably direction selective because during ND motion, the transient response to a test stimulus is even slightly enhanced in 9 of 13 recordings if a $24-\mathrm{Hz}$ stimulus is presented first (Fig. $6 B$ ). The time course of $\Delta\left[\mathrm{Ca}^{2+}\right]_{\mathrm{i}}$ during $24-\mathrm{Hz}$ motion (Fig. $6 B$, ...) is consistent with the finding that fast moving stimuli have only little influence on $\left[\mathrm{Ca}^{2+}\right]_{\mathrm{i}}$ (Dürr 1998). Nevertheless $\left[\mathrm{Ca}^{2+}\right]_{\mathrm{i}}$ increases that are hardly detectable by measuring $\Delta\left[\mathrm{Ca}^{2+}\right]_{\mathrm{i}}$ across the entire dendrite could be fairly large in close proximity to the cell membrane. Because such $\left[\mathrm{Ca}^{2+}\right]_{\mathrm{i}}$ increases could not be detected with our available imaging techniques, it appears at present most plausible to conclude that fast moving stimuli cause direction-selective adaptation by a mechanism that is probably independent of $\left[\mathrm{Ca}^{2+}\right]_{\mathrm{i}}$ increases.

\section{I S C U S S I O N}

Prolonged visual motion stimulation reduces the amplitude of a response to subsequent stimuli in directionally selective neurons of the fly. The effect, although present in both directions of motion, is much more pronounced and longer lasting for motion in the PD of the neuron. This direction selectivity is also observed in the after-reactions: an AHP, increasing in size with stimulus duration, is built up by PD motion, whereas comparable after-depolarizations are not elicited by ND motion. Adaptation and AHP could be based on the same physiological mechanism, for instance, a reduced excitatory or an increased inhibitory conductance. We propose dendritic $\mathrm{Ca}^{2+}$ as a physiological mediator of such a process. In our approach, direction selectivity of motion adaptation helps to discriminate mechanisms that are potentially based on $\mathrm{Ca}^{2+}$ from $\mathrm{Ca}^{2+}$. independent ones.

\section{Role of $\mathrm{Ca}^{2+}$ for the modulation of responsiveness}

When stimulus duration is varied, AHP and dendritic $\Delta\left[\mathrm{Ca}^{2+}\right]_{\mathrm{i}}$ are correlated in $\mathrm{HS}$ and $\mathrm{CH}$ cells (Fig. $4 C$ ). Although there are several possible mechanisms to cause an AHP, $\mathrm{Ca}^{2+}$ seems a likely candidate, given the evidence from other preparations. AHPs were shown to be mediated by $\mathrm{Ca}^{2+}$ dependent $\mathrm{K}^{+}$currents in a multitude of cell types (review: Sah 1996). This physiological mechanism is responsible for spike frequency adaptation in neurons (e.g., Chitwood and Jaffe 1998; Lo et al. 1998). Similarly, sensitivity changes in sensory cells are often mediated by ion channels that are directly or indirectly regulated by $\mathrm{Ca}^{2+}$ (reviews: Fettiplace and Fuchs
1999; Koutalos and Yau 1996; Menini 1999). For example, in vertebrate photoreceptors $\mathrm{Ca}^{2+}$ regulates sensitivity by both decreasing the affinity of the cGMP-gated cation channel for cGMP and inhibiting guanylate cyclase.

However, the correlation of the AHP and $\Delta\left[\mathrm{Ca}^{2+}\right]_{\mathrm{i}}$ observed in TCs does not prove that $\mathrm{Ca}^{2+}$ controls the AHP. To provide unequivocal evidence, it would be necessary to interfere effectively with $\left[\mathrm{Ca}^{2+}\right]_{\mathrm{i}}$ in TCs. Single (1998) reported an increase in the frequency of spike-like discharges superimposed on graded membrane potential responses by iontophoretic injection of the $\mathrm{Ca}^{2+}$ chelator 1,2-bis(o-aminophenoxy)ethane$N, N, N^{\prime}, N^{\prime}$-tetra-acetic acid (BAPTA) into the cytoplasm of TCs. This hints at a role of $\mathrm{Ca}^{2+}$ in the modulation of cellular excitability. In our own experiments, injection of BAPTA into single HS cells caused no significant change in AHP and adaptation. It has to be noted, however, that in vivo recording of TCs cannot yet be done with patch electrodes. Thus intracellular buffer concentrations cannot be controlled; something that would be necessary to rule out the existence of a BAPTAblockable, thus $\mathrm{Ca}^{2+}$-dependent, mechanism in motion adaptation.

Possible $\mathrm{Ca}^{2+}$-independent mechanisms leading to an AHP are for example slowly inactivating $\mathrm{K}^{+}$conductances, the $\mathrm{Na}^{+} / \mathrm{K}^{+}$pump (e.g., Gerster et al. 1997; Jansen and Nicholls 1973; Jansonius 1990) or $\mathrm{Na}^{+}$-dependent $\mathrm{K}^{+}$channels (e.g., Kim and McCormick 1998; Schwindt et al. 1989), the latter of which have been concluded to exist in blowfly HS neurons (Haag et al. 1997). Measuring the conductance change following motion stimulation and comparing its time course with that the AHP and of $\Delta\left[\mathrm{Ca}^{2+}\right]_{i}$ could help to determine the physiological mechanism underlying adaptation. However, due to ion channel kinetics and to putative differences in bulk cytosolic and near-membrane $\mathrm{Ca}^{2+}$ dynamics, the time course of a $\Delta\left[\mathrm{Ca}^{2+}\right]_{\mathrm{i}}$ is not necessarily identical to that of a $\mathrm{Ca}^{2+}$ dependent conductance (Jahromi et al. 1999; Sah and Clements 1999). Furthermore because different physiological mechanisms could cause similar conductance changes, an unequivocal identification of the conductance leading to AHP would additionally require pharmacological studies, which are beyond the scope of this study.

\section{Comparison of $\mathrm{HS}$ and $\mathrm{CH}$ cells}

In HS cells, the AHP and dendritic $\Delta\left[\mathrm{Ca}^{2+}\right]_{\mathrm{i}}$ are correlated, regardless of whether stimulus strength is altered by varying the duration or the temporal frequency of motion. $\mathrm{CH}$ cells, on the other hand, exhibit a monotonic relationship between AHP and $\Delta\left[\mathrm{Ca}^{2+}\right]_{\mathrm{i}}$ only for increases in stimulus duration but not for changes in temporal frequency (Figs. $4 C$ and 5). This is reminiscent of the finding that the temporal frequency tuning of electrical and $\mathrm{Ca}^{2+}$ responses is similar in HS cells but different in CH cells (Dürr 1998). Clearly, $\left[\mathrm{Ca}^{2+}\right]_{\mathrm{i}}$ is not the only determinant of the AHP in CH cells because AHPs were also present following ND stimuli (Fig. 4B). Further differences in motion-induced $\Delta\left[\mathrm{Ca}^{2+}\right]_{\mathrm{i}}$ between $\mathrm{HS}$ and $\mathrm{CH}$ cells, as found with respect to the time course and the spatial spread of $\mathrm{Ca}^{2+}$ accumulation, and the efficacy of ND stimuli in eliciting $\mathrm{Ca}^{2+}$ decrements have been found (Dürr 1998; Dürr and Egelhaaf 1999). At present it is unclear to what extent these differences correspond to cell-class-specific properties of motion adaptation, e.g., with respect to its temporal and spatial characteris- 
tics. So far, the differences between cell classes have been discussed to reflect a purely modulatory role of $\mathrm{Ca}^{2+}$ in $\mathrm{HS}$ cells and a putative involvement in transmitter release at the dendrodendritic synapses of $\mathrm{CH}$ cells.

\section{Implications for concepts of motion adaptation in TCs}

In the past, only presynaptic changes have been discussed to cause motion adaptation in TCs of the fly. One possible mechanism, although controversially discussed, is a decrease of EMD delay time constants, which would shift the temporal frequency tuning of TCs to higher velocities (de Ruyter van Steveninck et al. 1986; but see also Harris et al. 1999a). Borst and Egelhaaf (1987) showed that adaptation cannot only be elicited by motion stimulation but also by temporal modulation of luminance (flicker). However, the fact that motion stimuli were more effective than flicker stimuli in causing motion adaptation can be interpreted as a first hint that more than a single mechanism underlies motion adaptation in TCs. Recently Harris et al. (1999b) provided evidence for a reduction of contrast gain in HS cells induced by presenting adapting motion in any direction, including orthogonal motion, but not by presenting flicker. The most plausible explanation for this observation is an adaptation mechanism located presynaptic to TCs.

Here we suggest a postsynaptic mechanism for motion adaptation. We show that motion adaptation is to a certain extent associated specifically with PD motion. The TC itself is a likely location for the physiological mechanisms associated with direction-selective adaptation because it is regarded as the stage of motion processing where full direction selectivity is computed from only weakly direction-selective inputs (Borst and Egelhaaf 1990; Borst et al. 1995; Egelhaaf et al. 1989; Single et al. 1997). However, direction-selective motion adaptation is not simply determined by the excitation of the TC because membrane potential responses exhibit much faster time courses compared with the buildup of adaptation and recovery from it. Rather, temporally integrating the membrane potential response would be necessary to predict the strength of adaptation. Due to the time course of its accumulation, $\mathrm{Ca}^{2+}$ could mediate such a computational function. Nevertheless direction-selective motion adaptation seems not to be caused exclusively by a $\mathrm{Ca}^{2+}$-dependent mechanism. Fast-moving stimuli are shown to produce adaptation in a direction-selective manner without leading to considerable $\mathrm{Ca}^{2+}$ accumulation, thus providing further evidence for a heterogeneous origin of motion adaptation.

\section{Comparison with motion-sensitive neurons in other species}

Motion adaptation and its underlying mechanisms have also been investigated in motion-sensitive neurons of other species. Additionally, psychophysical studies (e.g., Clifford and Langley 1996b; Clifford and Wenderoth 1999) and functional magnetic resonance imaging (Tootell et al. 1995) have been performed on man to elucidate the nature of motion adaptation and its connection with the well-studied motion aftereffect. In this psychophysical phenomenon, prolonged viewing of a pattern moving in one direction results in illusory perception of movement in the opposite direction after the stimulus stops (review: Sekuler et al. 1978). In a direction-selective neuron such as a HS or $\mathrm{CH}$ cell, ND motion is signaled by membrane hyperpolarization. An AHP following prolonged PD motion might be "misinterpreted" by a subsequent processing stage as ND motion and could thus serve as a physiological explanation of the motion aftereffect.

The first physiological study on motion adaptation was by Barlow and Hill (1963) in the retinae of rabbits. They found that direction-selective ganglion cells exhibit a drop of discharge frequency below the spontaneous rate when movement stops after a long-lasting stimulation period.

Maddess et al. (1991) report that adaptation induces a shift of the velocity response curve toward higher velocities in motion-sensitive neurons of the butterfly Papilio aegeus. They propose the idea of piece-wise linearity, i.e., neurons responding linearly to changes in stimulus strength within each adaptation range. Similar changes in the temporal frequency tuning were reported for directional neurons in the nucleus of the optic tract (NOT) of the wallaby Macropus eugenii (Ibbotson et al. 1998). Adaptation, as assessed by determining decay time constants of responses to step-wise grating displacements (impulsive stimuli), was pronounced for PD motion but only weak with adapting stimuli moving in the ND or modulating temporally in luminance.

In the visual cortex of cats (area 17), adaptation also altered the temporal frequency tuning of motion-sensitive neurons, but instead of shifting the response curves, the strongest aftereffect occurred at the adapting frequency, with smaller effects both above and below it (Saul and Cynader 1989). This result is difficult to reconcile with a model incorporating adaptationinduced changes of time constants in EMDs unless there exist several kinds of EMDs with different delay filters. Also in cat visual cortex, Giaschi et al. (1993) report that in simple cells two processes are evident in the time courses of both the decrement of responsiveness during PD adaptation and its subsequent recovery: a rapid direction-independent and an additional slow, direction-selective process. This is very similar to the results on motion adaptation in fly TCs as described in the present study. Several further studies also show direction selectivity of motion adaptation in cat visual cortex (e.g., Hammond et al. 1988; Marlin et al. 1988) and area MT of the owl monkey (Petersen et al. 1985). However, the meaning of direction selectivity of adaptation in their and in our study is not completely congruent because most of the cortical neurons investigated are not fully direction selective in a strict sense. When stimulated with ND motion, the cortical neurons show weak response increments or no responses rather than decrements below resting activity as is found in fly TCs. Along with the different response characteristics of motion-sensitive cells, there probably exists a variety of mechanisms mediating motion adaptation. The present study presents evidence for a postsynaptic mechanism, namely dendritic $\mathrm{Ca}^{2+}$ accumulation, which can account for several features of motion adaptation in TCs of the fly, such as its direction selectivity, time course, and local restriction.

We thank B. Kimmerle for useful discussions of this work and R. Feiler for support in the programming of the $\mathrm{A} / \mathrm{D}$ converter. The constructive comments of two anonymous referees are gratefully acknowledged.

This work was supported by the Deutsche Forschungsgemeinschaft. 
Present address of V. Dürr: Abteilung für biologische Kybernetik und theoretische Biologie, Fakultät für Biologie, Universität Bielefeld, Postfach 10 01 31, D-33501 Bielefeld, Germany.

\section{REFERENCES}

BARLOW HB AND HiLl RM. Evidence for a physiological explanation of the waterfall phenomenon and figural after-effects. Nature 200: 1345-1347, 1963.

Borst A and EgelhaAf M.Temporal modulation of luminance adapts time constant of fly movement detectors. Biol Cybern 56: 209-215, 1987.

Borst A and EgelhaAf M. Principles of visual motion detection. Trends Neurosci 12: 297-306, 1989.

Borst A AND EgelhaAf M. Direction selectivity of blowfly motion-sensitive neurons is computed in a two-stage process. Proc Natl Acad Sci USA 87: 9363-9367, 1990.

Borst A AND EgelhaAf M. In vivo imaging of calcium accumulation in fly interneurons as elicited by visual motion stimulation. Proc Natl Acad Sci USA 89: 4139-4143, 1992.

Borst A, EgelhaAf M, and HaAg J. Mechanisms of dendritic integration underlying gain control in fly motion-sensitive interneurons. $J$ Comput Neurosci 2: 5-18, 1995.

Chitwood RA AND JAFFE DB. Calcium-dependent spike-frequency accomodation in hippocampal CA3 nonpyramidal neurons. J Neurophysiol 80: 983-988, 1998.

Clifford CWG and LANGley K. A model of temporal adaptation in fly motion vision. Vision Res 36: 2595-2608, 1996a.

CLIFFORD CWG AND LANGLEY K. Psychophysics of motion adaptation parallels insect electrophysiology. Curr Biol 6: 1340-1342, 1996b.

Clifford CWG and Wenderoth P. Adaptation to temporal modulation can enhance differential speed sensitivity. Vision Res 39: 4324-4332, 1999.

De Ruyter van Steveninck RR, ZaAgman WH, and Mastebroek HaK. Adaptation of transient responses of a movement-sensitive neuron in the visual system of the blowfly Calliphora erythrocephala. Biol Cybern 54: 223-236, 1986.

DÜRR V. Dendritic Calcium Accumulation in Visual Interneurons of the Blowfly (PhD dissertation). Bielefeld, Germany: University of Bielefeld, 1998.

DÜRR V AND EgElHaAF M. In vivo calcium accumulation in presynaptic and postsynaptic dendrites of visual interneurons. J Neurophysiol 82: $3327-$ 3338, 1999.

ECKERT H AND DVORAK DR. The centrifugal horizontal cells in the lobula plate of the blowfly, Phaenicia sericata. J Insect Physiol 29: 547-560, 1983.

EgelHAAF M AND Borst A. Transient and steady-state response properties of movement detectors. J Opt Soc Am A 6: 116-127, 1989.

EGELHAAF M AND BorsT A. A look into the cockpit of the fly: visual orientation, algorithms, and identified neurons. J Neurosci 13: 4563-4574, 1993a.

EgelhaAf M AND Borst A. Movement detection in arthropods. In: Visual Motion and its Role in the Stabilization of Gaze, edited by Miles MA and Wallman J. Amsterdam: Elsevier, 1993b, p. 53-77.

EGELHAAF M AND BORST A. Calcium accumulation in visual interneurons of the fly: stimulus dependence and relationship to membrane potential. $\mathrm{J} \mathrm{Neu}$ rophysiol 73: 2540-2552, 1995.

EgelhaAf M, Borst A, AND Reichardt W. Computational structure of a biological motion-detection system revealed by local detector analysis in the fly's nervous system. J Opt Soc Am A 6: 1070-1087, 1989.

EgelhaAf M, Borst A, Warzecha AK, Flecks S, and Wildemann A. Neural circuit tuning fly visual neurons to motion of small objects. II. Input organization of inhibitory circuit elements revealed by electrophysiological and optical recording techniques. J Neurophysiol 69: 340-351, 1993.

FetTiPlace R And Fuchs PA. Mechanisms of hair cell tuning. Annu Rev Physiol 61: 809-834, 1999.

Gauck V, EgelhaAf M, and Borst A. Synapse distribution on VCH, an inhibitory, motion-sensitive interneuron in the fly visual system. J Comp Neurol 381: 489-499, 1997.

Gerster U, Stavenga DG, and Backhaus W. $\mathrm{Na}^{+} / \mathrm{K}^{+}$-pump activity in photoreceptors of the blowfly Calliphora: a model analysis based on membrane potential measurements. J Comp Physiol [A] 180: 113-122, 1997.

Giaschi D, Douglas R, Marlin S, and Cynader M. The time course of direction-selective adaptation in simple and complex cells in cat striate cortex. J Neurophysiol 70: 2024-2034, 1993.
HaAg J, Theunissen F, and Borst A. The intrinsic electrophysiological characteristics of fly lobula plate tangential cells. II. Active membrane properties. J Comp Neurosci 4: 349-369, 1997.

Hammond P, Mouat GSV, and SMith AT. Neural correlates of motion after-effects in cat striate cortical neurones: monocular adaptation. Exp Brain Res 72: 1-20, 1988

HARRIS RA, O'CARROLL DC, AND LAUGHLIN SB. Adaptation and the temporal delay filter of fly motion detectors. Vision Res 39: 2603-2613, 1999a.

HARRIS RA, O'CARROLl DC, AND LAUGHLIN SB. Direction-independent component in fly motion adaptation. Proc Göttingen Neurobiol Conf 27: 434, 1999b.

HAUSEN K. Functional characterization and anatomical identification of motion sensitive neurons in the lobula plate of the blowfly Calliphora erythrocephala. Z Naturforsch [C] 31: 629-633, 1976.

HAUSEN K. Monocular and binocular computation of motion in the lobula plate of the fly. Verh Dtsch Zool Ges 1981: 49-70, 1981.

HAUSEN K. Motion sensitive interneurons in the optomotor system of the fly. I. The horizontal cells: structure and signals. Biol Cybern 45: 143-156, 1982a

HaUSEN K. Motion sensitive interneurons in the optomotor system of the fly. II. The horizontal cells: receptive field organization and response characteristics. Biol Cybern 46: 67-79, 1982b.

HAUSEN K. The lobula-complex of the fly: structure, function and significance in visual behaviour. In: Photoreception and Vision in Invertebrates, edited by Ali MA. New York: Plenum, 1984, p. 523-555.

HAUSEN K AND EgELHAAF M. Neural mechanisms of visual course control in insects. In: Facets of Vision, edited by Stavenga DG and Hardie RC. Berlin: Springer, 1989, p. 391-424.

IBBOTSON MR, CliFFORD CWG, AND MARK RF. Adaptation to visual motion in directional neurons of the nucleus of the optic tract. J Neurophysiol 79: 1481-1493, 1998.

Jahromi BS, Zhang L, Carlen PL, and Pennefather P. Differential timecourse of slow afterhyperpolarizations and associated $\mathrm{Ca}^{2+}$ transients in rat CA1 pyramidal neurons: further dissociation by $\mathrm{Ca}^{2+}$ buffer. Neuroscience 88: 719-726, 1999.

JANSEN JKS AND NiCHOLLS JG. Conductance changes, an electrogenic pump and the hyperpolarization of leech neurons following impulses. $J$ Physiol (Lond) 229: 635-655, 1973

JANSONIUS NM. Properties of the sodium pump in the blowfly photoreceptor cell. J Comp Physiol [A] 167: 461-467, 1990.

JiAN S AND HorRIDGE GA. The H1 neuron measures change in velocity irrespective of contrast frequency, mean velocity or velocity modulation frequency. Philos Trans R Soc Lond B Biol Sci 331: 205-211, 1991.

KIM U AND McCormick DA. Functional and ionic properties of a slow afterhyperpolarization in ferret perigeniculate neurons in vitro. $J$ Neurophysiol 80: 1222-1235, 1998.

Koutalos Y and YAU KW. Regulation of sensitivity in vertebrate rod photoreceptors by calcium. Trends Neurosci 19: 73-81, 1996.

KRAPP HG AND HENGSTENBERG R. Estimation of self-motion by optic flow processing in single visual interneurons. Nature 384: 463-466, 1996.

Lo FS, CORK RJ, AND Mize RR. Physiological properties of neurons in the optic layer of the rat's superior colliculus. J Neurophysiol 80: 331-343, 1998.

MadDESS T, Dubois RA, AND IBbotson MR. Response properties and adaptation of neurones sensitive to image motion in the butterfly Papilio aegeus. J Exp Biol 161: 171-199, 1991.

MADDESS T AND LAUGHLIN SB. Adaptation of the motion-sensitive neuron H1 is generated locally and governed by contrast frequency. Proc $R$ Soc Lond B Biol Sci 225: 251-275, 1985.

Marlin SG, Hasan SJ, AND Cynader MS. Direction-selective adaptation in simple and complex cells in cat striate cortex. J Neurophysiol 59: 13141330, 1988

MENINI A. Calcium signalling and regulation in olfactory neurons. Curr Opin Neurobiol 9: 419-426, 1999.

Petersen SE, BaKer JF, AND Allman JM. Direction-specific adaptation in area MT of the owl monkey. Brain Res 346: 146-150, 1985.

SAH P. $\mathrm{Ca}^{2+}$-activated $\mathrm{K}^{+}$currents in neurones: types, physiological roles and modulation. Trends Neurosci 19: 150-155, 1996.

SAH P AND ClemenTS JD. Photolytic manipulation of $\left[\mathrm{Ca}^{2+}\right]_{\mathrm{i}}$ reveals slow kinetics of potassium channels underlying the afterhyperpolarization in hippocampal pyramidal neurons. J Neurosci 19: 3657-3664, 1999.

SAUL AB AND CYNADER MS. Adaptation in single units in visual cortex: the tuning of aftereffects in the temporal domain. Vis Neurosci 2: 609-620, 1989. 
SChwindt PC, SpaIN WJ, AND CRILL WE. Long-lasting reduction of excitability by a sodium-dependent potassium current in cat neocortical neurons. J Neurophysiol 61: 233-244, 1989.

Sekuler R, Pantle A, and Levinson E. Physiological basis of motion perception. In: Handbook of Sensory Physiology. Perception, edited by Held R. Berlin: Springer, 1978, vol. VIII, p. 67-98.

SINGLE S. Mechanismen und Funktion dendritischer Kalziumsignale in visuellen Interneuronen der Fliege ( $\mathrm{PhD}$ dissertation). Tübingen, Germany: University of Tübingen, 1998.

SINGLE S AND BoRST A. Dendritic integration and its role in computing image velocity. Science 281: 1848-1850, 1998.

Single S, HAAG J, AND Borst A. Dendritic computation of direction selectivity and gain control in visual interneurons. J Neurosci 17: 6023-6030, 1997.

SRINIVASAn MV, Poteser M, AND Kral K. Motion detection in insect orientation and navigation. Vision Res 39: 2749-2766, 1999.
Tootell RBH, Reppas JB, Dale AM, Look RB, Sereno Mi, Malach R, BRADY TJ, AND RoSEN BR. Visual motion aftereffect in human cortical area MT revealed by functional magnetic resonance imaging. Nature 375: 139 $141,1995$.

VAUTIN RG AND BERKLEY MA. Responses of single cells in cat visual cortex to prolonged stimulus movement: neural correlates of visual aftereffects. J Neurophysiol 40: 1051-1065, 1977.

VRANESIC I AND KNÖPFEL T. Calculation of calcium dynamics from single wavelength fura-2 fluorescence recordings. Pflügers Arch Arch 418: 184$189,1991$.

Warzecha AK, EgelhaAf M, AND Borst A. Neural circuit tuning fly visual interneurons to motion of small objects. I. Dissection of the circuit by pharmacological and photoinactivation techniques. J Neurophysiol 69: 329339, 1993. 